\title{
Detail-Preserving Restoration of Impulse Noise Corrupted Images by a Switching Median Filter Guided by a Simple Neuro-Fuzzy Network
}

\author{
M. Emin Yüksel \\ Digital Signal and Image Processing Laboratory, Department of Electronics Engineering, Erciyes University, Kayseri 38039, Turkey \\ Email:yuksel@erciyes.edu.tr

\begin{abstract}
Alper Baştürk
Digital Signal and Image Processing Laboratory, Department of Electronics Engineering, Erciyes University, Kayseri 38039, Turkey Email: ab@erciyes.edu.tr
\end{abstract}

\section{Erkan Beşdok}

Department of Photogrammetry Engineering, Erciyes University, Kayseri 38039, Turkey

Email: ebesdok@erciyes.edu.tr

Received 25 August 2003; Revised 28 December 2003

\begin{abstract}
A new operator for the restoration of digital images corrupted by impulse noise is presented. The proposed operator is a simple recursive switching median filter guided by a neuro-fuzzy network functioning as an impulse detector. The internal parameters of the neuro-fuzzy impulse detector are adaptively optimized by training. The training is easily accomplished by using simple artificial images that can be generated in a computer. The most distinctive feature of the proposed operator over other operators is that it offers excellent detail- and texture-preservation performance, while effectively removing noise from the input image. Extensive experiments show that the proposed operator may be used for efficient restoration of digital images corrupted by impulse noise without distorting the useful information in the image.
\end{abstract}

Keywords and phrases: image processing, noise filtering, median filter, neuro-fuzzy systems.

\section{INTRODUCTION}

Digital images are valuable and important sources of information in many research and application areas including astronomy, biology, medicine, remote sensing, materials science, and so on $[1,2]$. During image acquisition and/or transmission, digital images are often contaminated by impulse noise due to a number of nonidealities in the imaging process. The noise is usually caused by either an imperfect medium between the original scene and the imaging system (random scattering and absorption) or a nonideal imaging system (sensor noise, limited system accuracy, finite precision and quantization of image data, etc.). The noise usually corrupts images by replacing some of the pixels of the original image with new pixels having luminance values near or equal to the minimum or maximum of the allowable dynamic luminance range.

In most applications, it is very important to remove impulse noise from image data, since the performances of subsequent image processing tasks are strictly dependent on the success of image noise removal operation. However, this is a difficult problem in any image processing system because the restoration filter must not distort the useful information in the image and preserve image details and texture while removing the noise. Conventional noise cancellation filters usually have the drawback of introducing undesirable distortions and blurring effects into the output image during noise cancellation process $[1,2]$.

A large number of methods have been proposed to remove impulse noise from digital images $[1,2,3,4,5,6,7,8$, $9,10,11,12,13,14,15,16,17,18,19]$. The majority of these methods comprise order statistic filters utilizing the rankorder information of an appropriate set of noisy input pixels. These filters are usually developed in the general framework of rank selection filters, which are nonlinear operators constrained to output an order statistic from a set of input samples. The difference between these filters is in the information used to decide which order statistic to output. 
The standard median filter [1] is a simple rank selection filter that attempts to remove impulse noise by changing the luminance value of the center pixel of the filtering window with the median of the luminance values of the pixels contained within the window. Although the median filter is simple and provides a reasonable noise removal performance, it removes thin lines and blurs image details even at low noise densities. The weighted median filter [3] and the center-weighted median filter [4] are modified median filters that give more weight to the appropriate pixels of the filtering window. These filters have been proposed to avoid the inherent drawbacks of the standard median filter by controlling the tradeoff between the noise suppression and detail preservation. They exhibit better performance in preserving image details than the median filter at the expense of reduced noise suppression performance. A method for the optimal design of weighted order statistic filters is also presented [5].

The standard and the weighted median filters are spatially invariant operators that are incapable of making any distinction between the corrupted and uncorrupted pixels of the noisy image. Hence, these filters distort the uncorrupted regions of the image during the restoration of the corrupted regions, causing the useful information in the image to be lost. The most straightforward solution to this problem is the switching median filter, which is obtained by combining the median filter with an impulse detector. In this approach, the impulse detector aims to determine whether the center pixel of a given filtering window is corrupted or not. If the center pixel is identified by the detector as a corrupted pixel, then it is replaced with the output of the median filter. Otherwise, it is left unchanged. Although this approach improves the performance of the median filter and reduces its distortion effects, its performance is dependent on the performance of the impulse detector. As a consequence, various switching median filters exploiting different impulse detection approaches based on median filters [6], multiple center-weighted median filters with different center weights [7], and edge detection kernels [8], have been proposed.

Some extensions of the basic switching median filter including multiple median-based filters in the structure have also been proposed. The tristate median filter [9] is an improved switching median filter that is obtained by adding a center-weighted median filter into the basic switching median filter structure. The multistate median filter (MSMF) [10] is a further extended version of the tristate median filter, including multiple center-weighted median filters. These two filters exhibit enhanced filtering performance at the expense of increased computational complexity.

The progressive switching median filter (PSMF) [11] is a derivative of the basic switching median filter. In this filtering approach, detection and removal of impulse noise are iteratively done in two separate stages. The filter provides more improved filtering performance than many other medianbased filters, but it has a very high computational complexity due to its iterative nature.

Signal-dependent rank-ordered mean filter (SDROMF) [12] is another switching filter utilizing rank-order information for impulse noise removal. The structure of the fil- ter is the same as a switching median filter except that the median filter is replaced with a rank-ordered mean filter. The SDROMF has been shown to exhibit significantly better noise removal and detail preservation performance than some conventional and state-of-the-art impulse noise filters for both grey scale [12] and colored [13] images. The minimum/maximum exclusive mean filter [14] is another type of switching rank-ordered mean filters. The filter demonstrates good filtering performance at the cost of increased computational complexity.

All of the above-mentioned methods more or less have the drawback of introducing undesirable distortions into the details and texture of the input image during noise removal process. Moreover, their performances depend on one or more parameters (such as, noise density estimate, weighting factors, impulse detection thresholds, etc.) that are heuristically determined and externally supplied by the user. Hence, it is difficult to determine the optimal parameters that yield the best filtering performance for a given image and a given noise density since there is no analytical method for this purpose.

In the last few years, there has been a growing research interest in the applications of soft computing techniques, such as neural networks and fuzzy systems, to the problems in digital image processing $[15,16,17,18,19,20,21,22]$. Indeed, neuro-fuzzy (NF) systems offer the ability of neural networks to learn from examples and the capability of fuzzy systems to model the uncertainty which is inevitably encountered in noisy environments. Therefore, NF systems may be utilized as very powerful tools for the detection and/or removal of impulse noise from digital images, provided that appropriate network structures and processing strategies are employed.

Hence, a number of nonlinear impulse noise removal operators based on soft computing techniques have also been proposed. One important class of these operators is fuzzy filters based on fuzzy if-then and if-then-else rules $[15,19]$. These operators usually employ a set of fuzzy rules for the detection of the impulse noise in a given filtering window, and an appropriate inference mechanism for its removal. Although they offer relatively better noise removal performance than the median-based operators, they are inherently heuristic and the determination of the fuzzy rules may be quite complicated especially when the noise density is high. In order to overcome this difficulty, methods that allow the determination of the internal parameters of the fuzzy filter (FF) by training have also been presented [18]. However, the structures of these systems are much more complex and the required filtering window size is usually larger than other methods. In addition, multi-output network topologies and the use of two-dimensional membership functions even more increase the complexity of these systems.

In this paper, a novel operator for efficient restoration of digital images corrupted by impulse noise is presented. The proposed operator is a hybrid filter constructed by combining a recursive switching median filter with a simple NF network functioning as an impulse detector. The internal parameters of the NF impulse detector are adaptively optimized by training. The training is easily accomplished by using 


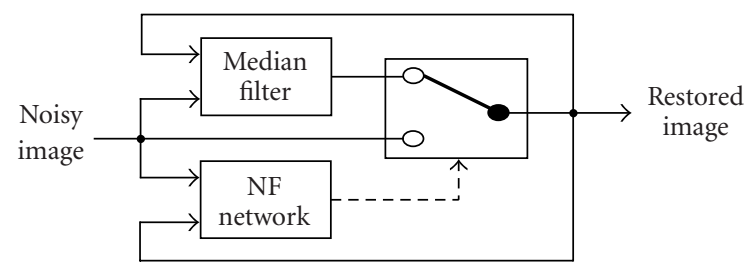

FIGURE 1: Structure of the proposed impulse noise removal operator. The operator is a recursive switching median filter guided by a simple neuro-fuzzy network functioning as an impulse detector.

simple artificial images that can be generated in a computer. Performance of the proposed filtering operator is tested at various noise densities and for different test images, and is also compared with conventional as well as state-of-the-art impulse noise removal operators. Experiments indicate that the new operator significantly outperforms the other operators and efficiently removes impulse noise from digital images without distorting image details and texture.

The rest of the paper is organized as follows. Section 2 explains the construction and training of the proposed operator. Section 3 discusses the implementation details of the proposed filter and its application to the test images. The experiments performed to evaluate the performance of the proposed filter and comparative interpretation of the results obtained are also discussed in this section. Section 4 , which is the final section, presents the conclusions and remarks.

\section{METHOD}

\subsection{The proposed operator}

Figure 1 shows the structure of the proposed noise removal operator. The operator is a hybrid filter obtained by appropriately combining a standard median filter, an NF network, and a switching mechanism in a recursive filtering structure. The NF network functions as an impulse detector and controls the operation of the switch. Both the median filter and the NF network operate recursively, that is, the output of the overall system is fed back to the inputs of both the median filter and the NF impulse detector. Therefore, the restored value of a given pixel is calculated by jointly utilizing the information from appropriate pixels of the noisy input and the restored output images.

The proposed operator processes the noisy input image pixel by pixel, starting from the upper left corner position, moving to the right and progressively downwards in a raster scanning fashion. The overall filtering procedure may be summarized as follows.

Let $x[r, c]$ denote the luminance value of a pixel at location $(r, c)$ of the noisy input image and $y[r, c]$ denote its restored value, that is, the output of the proposed operator. Here, $r$ and $c$ denote the row and column indices with $1 \leq r \leq R$ and $1 \leq c \leq C$ for an input image having a size of $R$-by- $C$ pixels.

For $r=1, \ldots, R$ and $c=1, \ldots, C$, the pixels of the restored output image are copied either from the correspond-

\begin{tabular}{|l|l|l|}
\hline$y[r-1, c-1]$ & $y[r-1, c]$ & $y[r-1, c+1]$ \\
\hline$y[r, c-1]$ & $x[r, c]$ & $x[r, c+1]$ \\
\hline$x[r+1, c-1]$ & $x[r+1, c]$ & $x[r+1, c+1]$ \\
\hline
\end{tabular}

Figure 2: A 3-by-3 pixel recursive filtering window centered at location $(r, c)$.

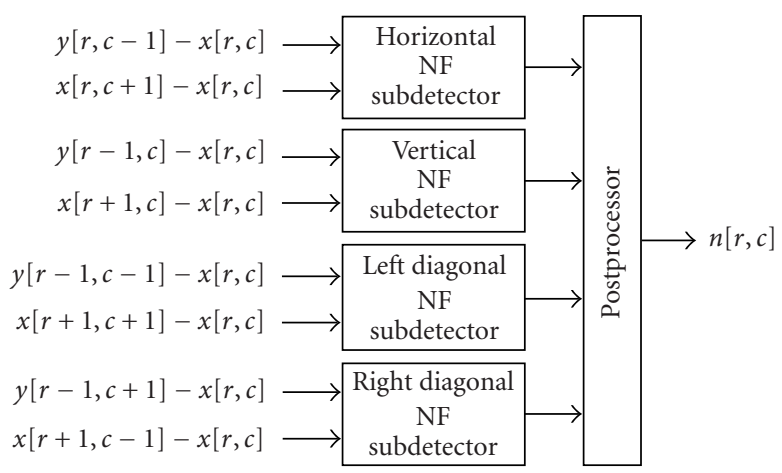

FIGURE 3: Structure of the neuro-fuzzy impulse detector. Each NF subdetector individually detects the presence of an impulse by evaluating pixel neighborhood in a different direction.

ing pixels of the input image or from the output of the median filter, depending on the output of the NF network,

$$
y[r, c]= \begin{cases}x[r, c] & \text { if } n[r, c]=0, \\ m[r, c] & \text { if } n[r, c]=1,\end{cases}
$$

where $n[r, c]$ is the output of the NF network, which can be either 0 or 1 , and $m[r, c]$ is the output of the median filter defined as

$$
m[r, c]=\operatorname{Median}\left(W_{N}[r, c]\right) .
$$

Here, $W_{N}[r, c]$ represents the set of pixels contained in a given recursive filtering window centered at location $(r, c)$ (see Figure 2.)

$$
W_{N}[r, c]=\{w[r+p, c+q] \mid(p, q) \in[-N, N]\},
$$

where $w[r+p, c+q]$ is defined as

$$
w[r+p, c+q]= \begin{cases}y[r+p, c+q] & \text { if } p<0 \text { or } q<0, \\ x[r+p, c+q] & \text { otherwise, }\end{cases}
$$

and $N$ is an integer number $(N \geq 1)$ related to the size of the filtering window, which is $(2 N+1)$-by- $(2 N+1)$ pixels. (For instance, $N=1$ for a 3-by-3 filtering window.)

\subsection{The impulse detector}

Figure 3 shows the structure of the NF network functioning as the impulse detector for the proposed operator. The network comprises four identical NF subdetectors and a postprocessor. The input data to the network are the differences 
between the luminance values of the center pixel of the filtering window and its eight neighbors. The filtering window is shown in Figure 2. The noisy input image is processed by moving the window on the image in a raster scanning fashion as discussed before, each time calculating the differences between the luminance values of the center pixel and its neighbors, and then feeding these difference values to the appropriate inputs of the four NF subdetectors, as illustrated in Figure 3.

Each subdetector individually attempts to detect the presence of an impulse at the center pixel of the filtering window by evaluating a specific pixel neighborhood in horizontal, vertical, left-diagonal, or right-diagonal direction, respectively. The outputs of the subdetectors are fed to a postprocessor, which makes the final decision about the presence of an impulse and controls the switch accordingly. The output of the postprocessor is equal to 1 if the center pixel of the filtering window is detected as an impulse, and 0 otherwise.

\subsection{The neuro-fuzzy subdetectors}

Each subdetector is a first-order Sugeno type fuzzy system with two inputs and one output [23]. Each input has three triangular type membership functions, whereas the output has a linear membership function. The inputoutput relationship of any of the four subdetectors is as follows.

Let $X_{1}$ and $X_{2}$ denote the inputs of the subdetector and $Y$ its output. Each possible combination of inputs and their associated membership functions is represented by a rule in the rule base of the subdetector. Since the subdetector has 2 inputs and each input has 3 membership functions, the rule base contains a total of $9\left(3^{2}\right)$ rules, which are as follows:

(1) if $\left(X_{1}\right.$ is $\left.M_{11}\right)$ and $\left(X_{2}\right.$ is $\left.M_{21}\right)$, then $R_{1}=F_{1}\left(X_{1}, X_{2}\right)$,

(2) if $\left(X_{1}\right.$ is $\left.M_{11}\right)$ and $\left(X_{2}\right.$ is $\left.M_{22}\right)$, then $R_{2}=F_{2}\left(X_{1}, X_{2}\right)$,

(3) if $\left(X_{1}\right.$ is $\left.M_{11}\right)$ and $\left(X_{2}\right.$ is $\left.M_{23}\right)$, then $R_{3}=F_{3}\left(X_{1}, X_{2}\right)$,

(4) if $\left(X_{1}\right.$ is $\left.M_{12}\right)$ and $\left(X_{2}\right.$ is $\left.M_{21}\right)$, then $R_{4}=F_{4}\left(X_{1}, X_{2}\right)$,

(5) if $\left(X_{1}\right.$ is $\left.M_{12}\right)$ and $\left(X_{2}\right.$ is $\left.M_{22}\right)$, then $R_{5}=F_{5}\left(X_{1}, X_{2}\right)$,

(6) if $\left(X_{1}\right.$ is $\left.M_{12}\right)$ and $\left(X_{2}\right.$ is $\left.M_{23}\right)$, then $R_{6}=F_{6}\left(X_{1}, X_{2}\right)$,

(7) if $\left(X_{1}\right.$ is $\left.M_{13}\right)$ and $\left(X_{2}\right.$ is $\left.M_{21}\right)$, then $R_{7}=F_{7}\left(X_{1}, X_{2}\right)$,

(8) if $\left(X_{1}\right.$ is $\left.M_{13}\right)$ and $\left(X_{2}\right.$ is $\left.M_{22}\right)$, then $R_{8}=F_{8}\left(X_{1}, X_{2}\right)$,

(9) if $\left(X_{1}\right.$ is $\left.M_{13}\right)$ and $\left(X_{2}\right.$ is $\left.M_{23}\right)$, then $R_{9}=F_{9}\left(X_{1}, X_{2}\right)$,

where $M_{i j}$ denotes the $j$ th membership function of the $i$ th input, $R_{k}$ denotes the output of the $k$ th rule, and $F_{k}$ denotes the $k$ th output membership function. The input membership functions are triangular type and defined as

$$
M_{i j}(u)= \begin{cases}0 & \text { if } u \leq a_{i j}, \\ \frac{u-a_{i j}}{b_{i j}-a_{i j}} & \text { if } a_{i j} \leq u \leq b_{i j}, \\ \frac{c_{i j}-u}{c_{i j}-b_{i j}} & \text { if } b_{i j} \leq u \leq c_{i j}, \\ 0 & \text { if } c_{i j} \leq u\end{cases}
$$

for $i=1,2 ; j=1,2,3$, or in a more compact form:

$$
\begin{aligned}
& M_{i j}(u) \\
& =\max \left(\min \left(\frac{u-a_{i j}}{b_{i j}-a_{i j}}, \frac{c_{i j}-u}{c_{i j}-b_{i j}}\right), 0\right), \quad i=1,2 ; j=1,2,3 .
\end{aligned}
$$

The output membership functions are linear and defined as

$$
F_{k}\left(u_{1}, u_{2}\right)=d_{k 1} u_{1}+d_{k 2} u_{2}+d_{k 3}, \quad k=1, \ldots, 9 .
$$

Here, the parameters $a, b, c$, and $d$ are constants that characterize the shape of the membership functions. The optimal values of these parameters are determined by training, which will be discussed in detail later on.

The output of the subdetector is the weighted average of the individual rule outputs. The weighting factor, $w_{k}$, of each rule is calculated by evaluating the membership expressions in the antecedent of the rule. This is accomplished by first converting the input values to fuzzy membership values by utilizing the input membership functions and then applying the and operator to these membership values. The and operator corresponds to the multiplication of input membership values. Hence, the weighting factors of the rules are calculated as follows:

$$
\begin{aligned}
& w_{1}=M_{11}\left(X_{1}\right) \cdot M_{21}\left(X_{2}\right), \\
& w_{2}=M_{11}\left(X_{1}\right) \cdot M_{22}\left(X_{2}\right), \\
& w_{3}=M_{11}\left(X_{1}\right) \cdot M_{23}\left(X_{2}\right), \\
& w_{4}=M_{12}\left(X_{1}\right) \cdot M_{21}\left(X_{2}\right), \\
& w_{5}=M_{12}\left(X_{1}\right) \cdot M_{22}\left(X_{2}\right), \\
& w_{6}=M_{12}\left(X_{1}\right) \cdot M_{23}\left(X_{2}\right), \\
& w_{7}=M_{13}\left(X_{1}\right) \cdot M_{21}\left(X_{2}\right), \\
& w_{8}=M_{13}\left(X_{1}\right) \cdot M_{22}\left(X_{2}\right), \\
& w_{9}=M_{13}\left(X_{1}\right) \cdot M_{23}\left(X_{2}\right) .
\end{aligned}
$$

Once the weighting factors are obtained, the output of the subdetector can be found by calculating the weighted average of the individual rule outputs:

$$
Y=\frac{\sum_{k=1}^{9} w_{k} R_{k}}{\sum_{k=1}^{9} w_{k}} .
$$

Readers interested in the details of fuzzy systems may refer to an excellent book on this subject [23].

\subsection{The postprocessor}

The outputs of the four NF subdetectors are fed to a postprocessor, which generates the final detector output. The postprocessor actually calculates the average value of the four subdetector outputs and then suitably maps this value to either 0 or 1 . This is accomplished by comparing the average value with a threshold, which is equal to the middle value of the allowable dynamic range for the luminance values of 


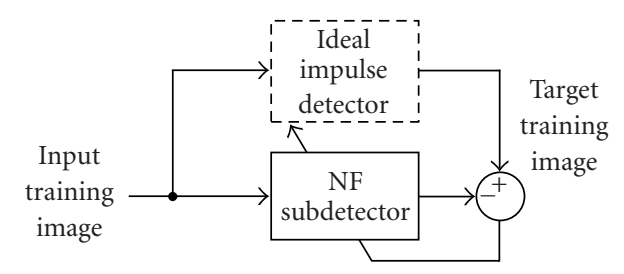

Figure 4: Training of the neuro-fuzzy subdetectors.

the pixels of the input image. The computing process of the postprocessor can be formulated as follows.

Let $Y_{H}, Y_{V}, Y_{L}$, and $Y_{R}$ represent the outputs of the four NF subdetectors, respectively. The output of the postprocessor, which is also the output of the NF impulse detector, is calculated as follows:

$$
n[r, c]= \begin{cases}0 & \text { if } Y_{\mathrm{AV}}<T, \\ 1 & \text { if } Y_{\mathrm{AV}} \geq T .\end{cases}
$$

Here, $Y_{\mathrm{AV}}$ is the average value of the four subdetector outputs:

$$
Y_{\mathrm{AV}}=\frac{Y_{H}+Y_{V}+Y_{L}+Y_{R}}{4}
$$

and $T$ is the threshold value, which is equal to the middle value of the allowable dynamic luminance range,

$$
T=\frac{L_{\min }+L_{\max }}{2},
$$

where $L_{\min }$ and $L_{\max }$ represent the minimum and the maximum values of the allowable dynamic luminance range, respectively. For 8-bit images, $L_{\min }$ is 0 and $L_{\max }$ is 255 .

\subsection{Training of the neuro-fuzzy subdetectors}

The internal parameters of the NF impulse detector are determined by training. Each NF subdetector in the network is individually trained. Figure 4 represents the arrangement used for training. Here, the parameters of the NF subdetector under training are iteratively tuned so that its output converges to the output of the ideal impulse detector which, by definition, can truly detect the presence of an impulse. The ideal impulse detector is only a conceptual detector and does not necessarily exist in reality. It is only the output of the ideal impulse detector that is needed for training, and this is represented by the target training image.

Figure 5 shows the images used for training. The image shown in Figure 5a is the base training image. This is a 128by-128 pixel artificial image and can easily be generated in a computer. Each square box in this image has a size of 4-by-4 pixels and the 16 pixels contained within each box have the same luminance value, which is a random integer number uniformly distributed in $[0,255]$.

The image in Figure $5 \mathrm{~b}$ is the input training image. This image is obtained by corrupting the base training image by impulse noise of $30 \%$ noise density. Although the density of the corrupting noise is not very critical regarding training performance, it is experimentally observed that the detector performs best when the noise density of the input training image is equal to the noise density of the noisy input image to be restored. It is also observed that the performance of the detector slightly decreases as the difference between the two noise densities increases. Since it is usually impossible to know the actual noise density of a corrupted image in a real practical application, very low and very high values for training noise density should be avoided in order to obtain a stable detection performance for a wide range of filtering noise densities. Results of extensive simulation experiments show that very good detection performance can easily be obtained for all kinds of images corrupted by impulse noise with a noise density between $0 \%$ and $80 \%$, provided that the input training image has a noise density between $20 \%$ and $50 \%$.

The image in Figure $5 \mathrm{c}$ is the target training image. This is a black-and-white image generated from the difference between the base and the input training images. Luminance values of its pixels are obtained by subtracting the luminance values of the pixels of the input training image from the luminance values of the corresponding pixels of the base training image, and then mapping the zero values to black pixels and the nonzero values to white pixels. Hence, the locations of the white pixels in the target training image indicate the locations of the noisy pixels in the input training image.

The images in Figures $5 b$ and $5 c$ are used as the input and the target (desired) images during training, respectively. The parameters of the NF subdetector under training are then iteratively adjusted by using the Levenberg-Marquardt optimization algorithm [23] so as to minimize the learning error. After finishing the training of all NF subdetectors, the internal parameters of the subdetectors are fixed and they are combined with the postprocessor, as shown in Figure 3, to construct the NF impulse detector.

\section{RESULTS}

The proposed operator discussed in the previous section is implemented. The performance of the operator is tested under various noise conditions and on several popular images from the literature including Baboon, Blood, Boats, Bridge, Cameraman, Goldhill, Lena, Pentagon, Peppers, and Rice. These images are shown in Figure 6. All images are 8-bit grey level images having the same size of 256-by-256 pixels. The test images used in the experiments are generated by contaminating the original images by impulse noise with an appropriate noise density depending on the experiment.

For comparison, the corrupted test images are also filtered by using several conventional and state-of-the-art impulse noise removal operators including the standard median filter (MF) [1], the edge-detecting median filter (EDMF) [8], MSMF [10], the PSMF [11], the SDROMF [12], and an FF [15]. These filters are representative implementations of different approaches to the impulse noise filtering problem. 


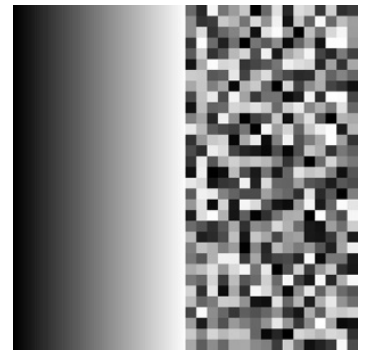

(a)

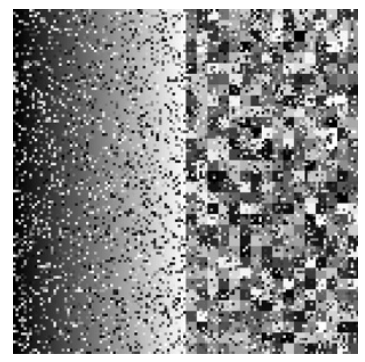

(b)

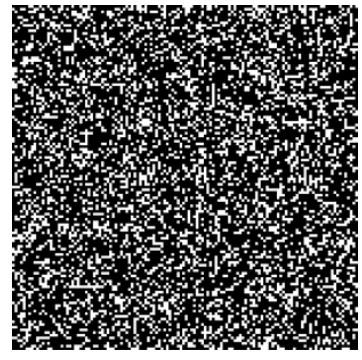

(c)

FIGURE 5: The training images: (a) base training image, (b) input training image, and (c) target training image.

The EDMF, MSMF, PSMF, SDROMF, and FF operators have a number of tuning parameters. Unfortunately, there is no analytical method to determine the optimal values for these parameters that yield the best results for a given filtering experiment. Hence, the values of these parameters are heuristically determined for each individual experiment. In the experiments presented in this paper, the values suggested in the corresponding references are used. These are as follows. For EDMF, $T=116$ [8]. For MSMF, $w_{\max }=5$ and $T=30$ [10]. For PSMF, $N_{D}=3$ and $N_{F}=3$ [11]. For SDROMF, $\alpha_{1}=0, \alpha_{2}=1$, and the thresholds $\left\{T_{1}, T_{2}, T_{3}, T_{4}\right\}$ are $\{8,20,40,50\}$ [12]. For FF, $L=256, a=40$, and $b=32$ [15]. All filters including the proposed filter operate on a 3-by-3 filtering window, except the EDMF filter which performs better with a 5-by-5 window [8].

The performances of all operators are evaluated by using the mean squared error (MSE) criterion, which is defined as

$$
\operatorname{MSE}=\frac{1}{R C} \sum_{r=1}^{R} \sum_{c=1}^{C}(s[r, c]-y[r, c])^{2},
$$

where $s[r, c]$ and $y[r, c]$ represent the original and the restored versions of a corrupted test image, respectively.

Several experiments are performed to measure and compare the noise suppression and detail preservation performances of all operators. The experiments are especially designed to reveal the performances of the operators for different image properties and noise conditions.

Since all experiments are related with noise and noise is a random process, every realization of the same experiment yields different results even if the experimental conditions are the same. Therefore, each individual filtering experiment presented in this paper is repeated for 10 times yielding 10 different MSE values for the same experiment. The average of these values is then taken as the representative MSE value for that experiment.

\subsection{Experiment set 1 (constant noise density)}

In the first set of experiments, the original images shown in Figure 6 are corrupted by impulse noise having a noise density of $25 \%$. The corrupted images are filtered by all operators and the MSE values of the output images are calculated.
In order to obtain a deeper analysis of the filtering behavior and to separately evaluate the noise suppression and detail preservation ability of the operators, the MSE values are separately calculated (i) for all pixels, (ii) for corrupted pixels only, and (iii) for uncorrupted pixels only. These are given in Tables 1, 2, and 3, respectively.

The MSE values listed in Table 1 are calculated for all pixels and reflect the overall filtering performances of the operators. As it is seen from this table, performance of the MF is the worst of all for all test images. The MSMF is considerably better than the MF but worse than the others. The performances of the EDMF, PSMF, SDROMF, and FF are very close to each other. The proposed filter, however, exhibits the best performance for all test images.

Table 2 lists the MSE values calculated for only the corrupted pixels of the test images for a comparative evaluation of the noise removal performances of the operators. This time the performance of the MF is better than the MSMF, as expected. This is because the MSMF is based on a certain number of center-weighted median filters with different center weights. As discussed in the Introduction, the centerweighted median filter demonstrates better detail preservation performance than the standard median filter at the cost of reduced noise removal performance. Hence, the noise removal performance of the MSMF is worse than that of the MF. The performances of the SDROMF, PSMF, and FF are again very close to each other, whereas the EDMF yields relatively higher MSE values. This is because the EDMF uses edge detection kernels to detect impulses. The decrease in the performance of this operator may be attributed to the false classification of a number of impulses as edge pixels. As a consequence, these pixels are left unfiltered, which results in a decrease in the noise suppression performance of the EDMF operator. On the other hand, the performance of the proposed filter is considerably better than the other filters for all images except the Pentagon image, for which the PSMF performs slightly better.

The real power of the proposed filter is revealed by Table 3, which lists the MSE values calculated for only the uncorrupted pixels of the test images for a comparative evaluation of the detail preservation performances of the operators. An ideal noise filter would only process the corrupted 


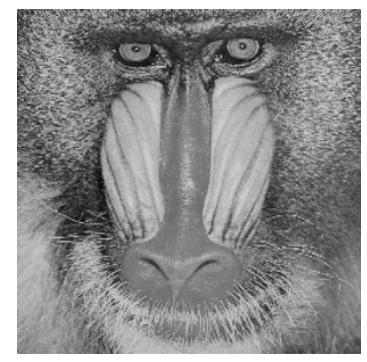

(a)

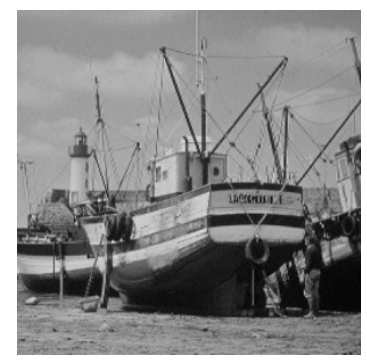

(c)

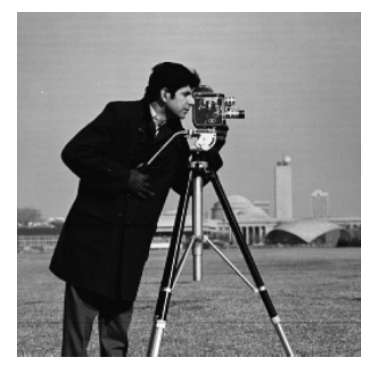

(e)

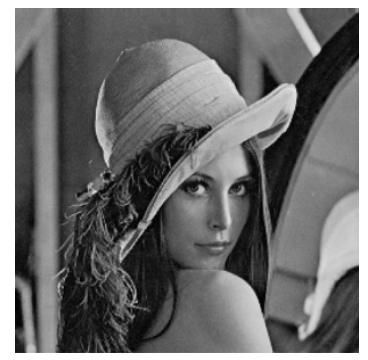

(g)

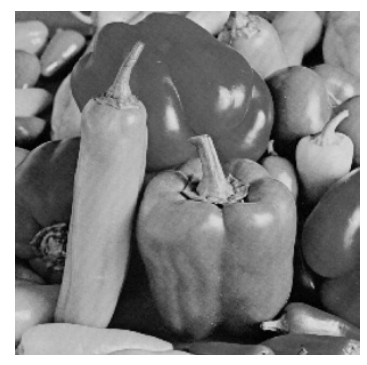

(i)

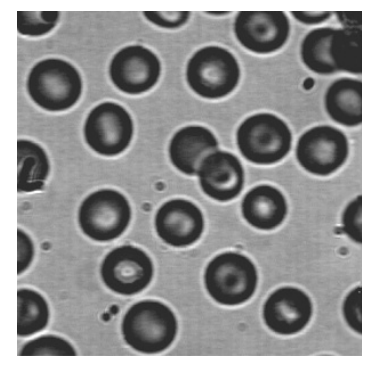

(b)

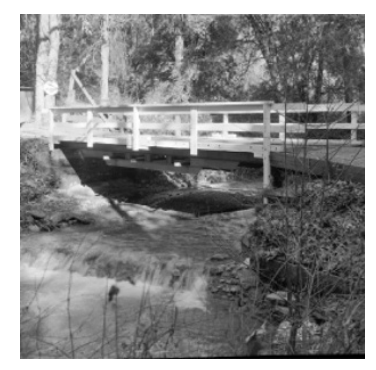

(d)

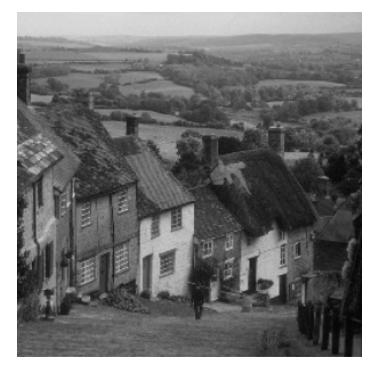

(f)

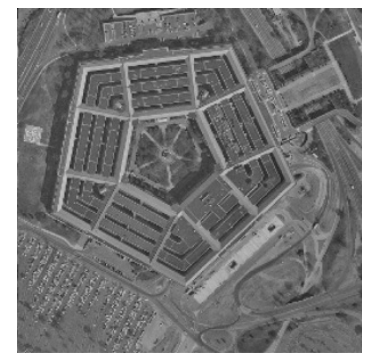

(h)

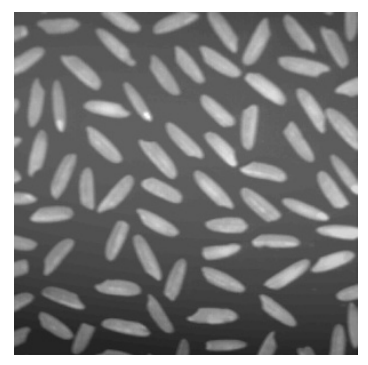

(j)
Figure 6: Test images: (a) Baboon, (b) Blood, (c) Boats, (d) Bridge, (e) Cameraman, (f) Goldhill, (g) Lena, (h) Pentagon, (i) Peppers, and (j) Rice. pixels of a noisy input image and leave the uncorrupted pixels unchanged. Therefore, the MSE value calculated for only the uncorrupted pixels of a given test image would be zero for an ideal noise filter. However, it is easily seen from Table 3 that the MSE values of all filters except the proposed filter are significantly higher than zero. This obviously implies that these operators significantly distort the uncorrupted regions of the test images during restoration of the corrupted regions, resulting in undesirable blurring effects in the details of the input image and loss of useful information within the image. On the other hand, the MSE values of the proposed operator are significantly lower than those of the other operators and much closer to zero. This clearly indicates that the filtering behavior of the proposed operator is much closer to that of an ideal filter regarding detail preservation when compared to the other filters. Hence, the proposed operator effectively preserves the useful information contained within the uncorrupted regions of the input image during restoration of the corrupted regions.

The output images of all operators are shown in Figure 7 for a visual evaluation of the noise removal and detail preservation performances of the operators. Due to space restrictions, the output images are shown only for the Baboon image corrupted by impulse noise with $25 \%$ noise density. This image is especially chosen for comparison because of its rich detail and texture. It is observed from this figure that the performance of the MF and MSMF are very close to each other. Some noise blotches are easily visible in the output images of these two filters. The output images of the SDROMF and FF are almost indistinguishable from each other and they are significantly better than those of the MF and MSMF. However, a few noise blotches are still visible. The PSMF shows superior noise removal performance but considerably distorts the details of the image. On the contrary, the EDMF exhibits much better detail preservation performance than the other operators at the cost of slightly reduced noise suppression performance. The proposed filter, however, demonstrates very good noise suppression and excellent detail preservation performance. The difference, especially in detail preservation, can easily be observed by carefully comparing the appearance of the eyes and the hair around the mouth of the animal in all images.

\subsection{Experiment set 2 (varying noise density)}

The second set of experiments are designed to evaluate the dependency of the performances of the operators on noise density. For this purpose, the noise density is increased from $5 \%$ to $90 \%$ with $5 \%$ steps. For each noise density step, the ten test images shown in Figure 6 are corrupted by impulse noise with the same step noise density. This produces ten different experimental images, each having the same noise density. These images are filtered by one of the operators and the MSE values are calculated for all pixels of the output images, producing ten different MSE values representing the overall filtering performance of that operator under different image properties. These values are then averaged to obtain the representative MSE value of that operator for that noise density. This procedure is separately repeated for all noise densities to 
TABLE 1: Comparison of the overall filtering performances of the operators. The test images are corrupted by 25\% impulse noise. The MSE values are calculated for all pixels of the input images.

\begin{tabular}{l|rrrrrrrrrrr}
\hline Operator & Baboon & Blood & Boats & Bridge & Cameraman & Goldhill & Lena & Pentagon & Peppers & Rice & Average \\
\hline Noisy & 4511 & 4754 & 4760 & 4825 & 5071 & 4741 & 4968 & 4345 & 4810 & 4597 & 4738 \\
MF & 800 & 228 & 373 & 382 & 380 & 301 & 294 & 393 & 253 & 157 & 356 \\
MSMF & 560 & 168 & 270 & 262 & 312 & 214 & 229 & 268 & 196 & 121 & 260 \\
EDMF & 455 & 152 & 267 & 236 & 294 & 192 & 182 & 212 & 167 & 81 & 224 \\
SDROMF & 569 & 94 & 233 & 222 & 292 & 154 & 171 & 227 & 134 & 42 & 214 \\
FF & 449 & 126 & 206 & 192 & 264 & 150 & 184 & 165 & 141 & 57 \\
PSMF & 533 & 119 & 281 & 206 & 241 & 132 & 165 & 189 & 128 & 40 & 203 \\
Proposed & 22 & 50 & 97 & 100 & 101 & 73 & 73 & 98 & 62 & 25 & 90 \\
\hline
\end{tabular}

TABle 2: Comparison of the noise suppression performances of the operators. The test images are corrupted by $25 \%$ impulse noise. The MSE values are calculated for only the corrupted pixels of the input images.

\begin{tabular}{l|rrrrrrrrrrr}
\hline Operator & Baboon & Blood & Boats & Bridge & Cameraman & Goldhill & Lena & Pentagon & Peppers & Rice & Average \\
\hline Noisy & 18058 & 18958 & 19056 & 19342 & 20280 & 18923 & 19848 & 17433 & 19253 & 18374 & 18953 \\
MF & 1181 & 473 & 651 & 669 & 681 & 556 & 549 & 651 & 496 & 344 & 625 \\
MSMF & 1311 & 644 & 861 & 876 & 994 & 739 & 772 & 769 & 717 & 476 & 816 \\
EDMF & 1025 & 430 & 658 & 642 & 646 & 512 & 493 & 568 & 435 & 260 & 567 \\
SDROMF & 932 & 246 & 473 & 489 & 550 & 349 & 382 & 419 & 319 & 119 & 428 \\
FF & 947 & 382 & 511 & 546 & 529 & 425 & 491 & 413 & 372 & 180 & 480 \\
PSMF & 892 & 373 & 552 & 498 & 489 & 390 & 453 & 383 & 360 & 146 & 454 \\
Proposed & 888 & 198 & 386 & 398 & 395 & 291 & 284 & 393 & 243 & 100 & 358 \\
\hline
\end{tabular}

TABLE 3: Comparison of the detail-preservation performances of the operators. The test images are corrupted by $25 \%$ impulse noise. The MSE values are calculated for only the uncorrupted pixels of the input images.

\begin{tabular}{l|crrrrrrrrrr}
\hline Operator & Baboon & Blood & Boats & Bridge & Cameraman & Goldhill & Lena & Pentagon & Peppers & Rice & Average \\
\hline Noisy & 0 & \multicolumn{1}{c}{0} & \multicolumn{1}{c}{0} & \multicolumn{1}{c}{0} & \multicolumn{1}{c}{0} & 0 & 0 & \multicolumn{1}{c}{0} & \multicolumn{1}{c}{0} & \multicolumn{1}{c}{0} & \multicolumn{1}{c}{0} \\
MF & 672.9 & 146.0 & 280.0 & 286.8 & 279.6 & 215.7 & 208.2 & 306.9 & 172.5 & 94.0 & 266.3 \\
MSMF & 309.3 & 8.9 & 72.6 & 58.3 & 84.8 & 39.1 & 47.6 & 101.5 & 22.5 & 3.1 & 74.8 \\
EDMF & 265.7 & 59.3 & 136.4 & 100.6 & 176.6 & 85.3 & 78.3 & 94.1 & 77.9 & 20.9 & 109.5 \\
SDROMF & 448.4 & 43.3 & 153.6 & 132.6 & 206.6 & 88.6 & 100.5 & 162.8 & 72.8 & 16.8 & 142.6 \\
FF & 283.3 & 39.8 & 104.9 & 74.1 & 175.2 & 58.6 & 81.0 & 82.9 & 64.1 & 15.7 & 98.0 \\
PSMF & 413.9 & 34.3 & 190.0 & 108.7 & 157.9 & 46.3 & 69.3 & 124.9 & 50.1 & 4.2 & 120.0 \\
Proposed & 0.7 & 0.6 & 0.1 & 0.9 & 2.9 & 0.0 & 2.5 & 0.1 & 2.0 & 0.2 & 1.0 \\
\hline
\end{tabular}

obtain the variation of the average MSE value of that operator as a function of noise density. Finally, the overall experimental procedure is individually repeated for each operator.

Figure 8 shows the variation of the average MSE values of the operators as a function of noise density. As it is seen from this figure, the performances of the MF and MSMF operators are very poor, with the MSMF being slightly better than the MF. The EDMF performs better than the MF and MSMF but worse than the others. As discussed in the previous subsection (Experiment set 1), the EDMF operator utilizes edge detection kernels to distinguish between corrupted, uncorrupted, and edge pixels. In this way, the smoothing effect of the filter, which is desirable for noisy pixels but undesirable for edge pixels, is suitably controlled. But, the fundamental drawback of this approach is that an increasing number of noisy pixels are incorrectly detected as edge pixels as the noise density increases. As a result, the performance of the EDMF operator relatively decreases with increasing noise density. This is observed in Figure 8. The performances of the SDROMF and FF operators are almost the same, with FF being slightly better for noise densities up to approximately $80 \%$. These operators exhibit considerably better filtering performance than the MF, MSMF, and EDMF operators for all noise densities. The filtering performance of the PSMF operator is better at relatively higher noise densities than at lower noise densities. As discussed in the previous subsection, this operator exhibits very good noise suppression performance, but significantly distorts image details and texture. As the noise density increases, the number of corrupted pixels in the input images increases. As a consequence, the contribution of the noise suppression performance to the general filtering performance becomes more dominant than the 


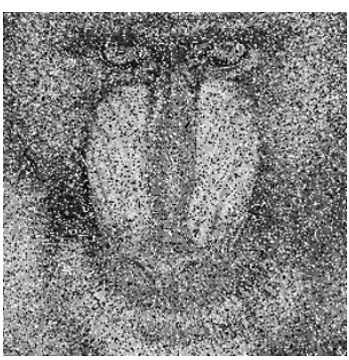

(a)

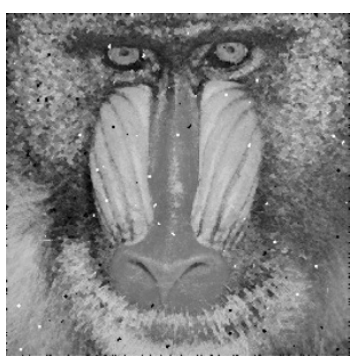

(c)

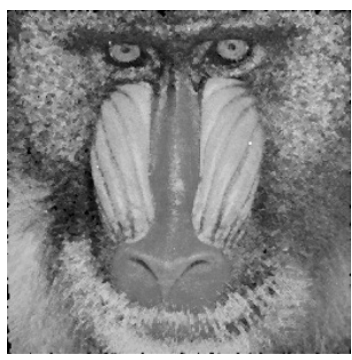

(e)

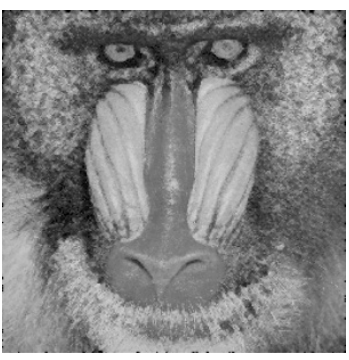

(g)

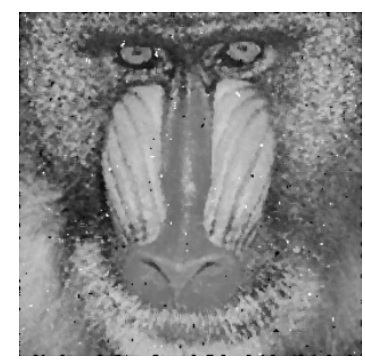

(b)

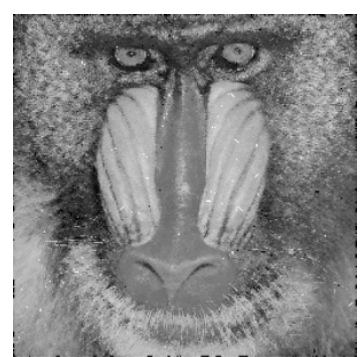

(d)

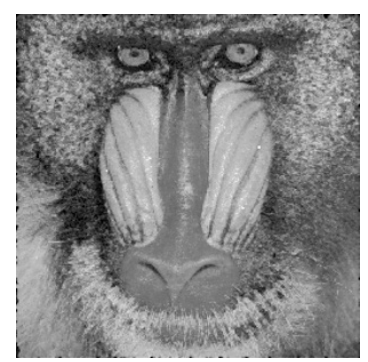

(f)

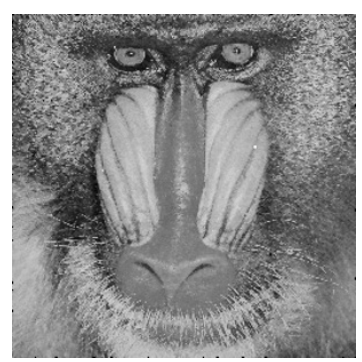

(h)
Figure 7: Output images of the operators for the Baboon image corrupted by impulse noise with $25 \%$ noise density: (a) noisy Baboon image, (b) MF, (c) MSMF, (d) EDMF, (e) SDROMF, (f) FF, (g) PSMF, and (h) proposed.

contribution of the detail preservation performance. Therefore, the performance of the PSMF operator becomes better than the other operators for relatively higher noise densities, as observed in Figure 8. The proposed operator, however, demonstrates the best filtering performance of all. Its MSE values are significantly lower than those of the other filters for all noise densities.

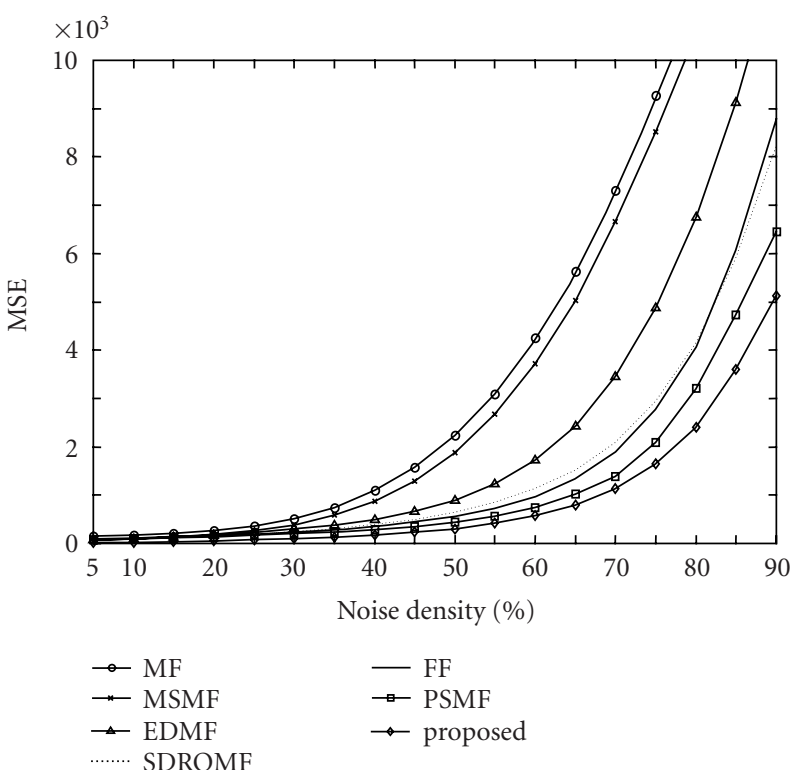

Figure 8: Performances of all operators as a function of noise density.

\subsection{Experiment set 3 (false alarm ratio)}

The experiments in this set are intended to provide a comparative evaluation of the false alarm ratio (FAR) values of the impulse detectors of the operators as a function of noise density. The MF operator is not included in these experiments, since it is not a switching filter and hence does not have an impulse detector.

The experimental procedure to obtain the variation of the FAR value for the impulse detector of a given operator is as follows. First, one of the original images shown in Figure 6 is chosen and this image is corrupted by impulse noise with noise densities increasing from $5 \%$ to $90 \%$ with $5 \%$ steps, producing 18 experimental images, each having a different noise density. Then, each of these experimental images are separately processed by the impulse detector of the given operator, and their pixels are classified as either corrupted or uncorrupted. Next, the variation of FAR as a function of noise density is obtained by individually calculating the FAR value of each of the experimental images by using the formula

$$
\text { FAR }=\frac{\text { number of incorrectly classified pixels }}{\text { total number of pixels }} \times 100 \text {. }
$$

Following this, the same experimental procedure is separately repeated for the remaining nine images in Figure 6. After that, the results are averaged to obtain the variation of the FAR for the impulse detector of the given operator. Finally, the overall experimental procedure is separately repeated for the other operators.

Figure 9 shows the variation of the FAR values as a function of noise density. It is seen that the FAR values of the 


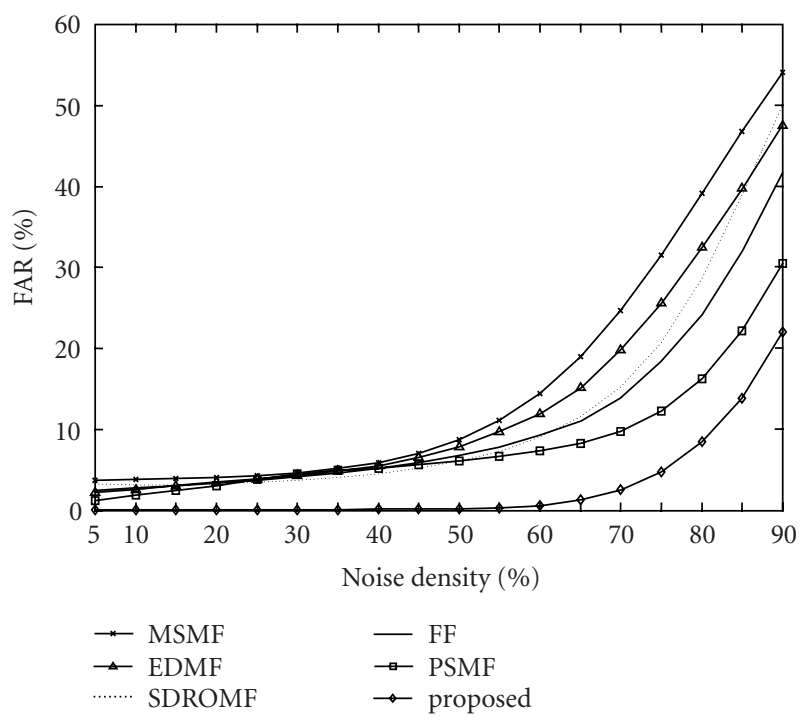

FIGURE 9: False alarm ratio of the proposed operator as a function of noise density.

MSMF, EDMF, SDROMF, FF, and PSMF operators are very close to and slightly different from each other for noise densities up to $20 \%$. For noise densities between $20 \%$ and $40 \%$, the FAR values of these operators are almost the same, indicating that they exhibit almost the same detection performance. The performance difference becomes more apparent as the noise density increases from $40 \%$ to higher. For noise densities higher than around 50\%, the FAR values of these operators rapidly increase as the noise density increases.

The impulse detector of the proposed filter performs very well and its FAR values are much lower than those of the other operators for all noise densities. Especially for noise densities up to $60 \%$, the FAR values of the proposed filter are very low, which implies that the NF network correctly classifies a great majority of the pixels of the noisy input image. This is actually the main reason of the excellent detail preservation capability of the proposed filter and confirms the results presented in Table 3 . As the noise density increases, the FAR value of the proposed filter also increases as it is naturally expected. However, it is seen that the FAR values of the proposed filter remain much less than those of the other filters for all noise densities, resulting in superior filtering performance.

\section{DISCUSSION AND CONCLUSION}

A novel operator for removing impulse noise from digital images is presented. The fundamental superiority of the proposed operator over other operators is that it efficiently removes impulse noise from digital images while successfully preserving the details and texture in the original image. The advantages of the new operator over other operators in the literature may be summarized as follows.
(1) It has a very simple structure. It is constructed by appropriately combining a median filter and an NF network. The structure of the NF network is also very simple. Its fundamental building block is a first-order Sugeno type fuzzy system with two inputs and one output.

(2) The NF network uses one-dimensional fuzzy membership functions, which simplifies implementation. On the other hand, some NF systems related to impulse noise filtering utilize two-dimensional fuzzy membership functions [18].

(3) It operates on a 3-by-3 filtering window, whereas some other operators in the literature require filtering windows with larger window sizes $[8,18]$. The larger the size of the filtering window, the higher the number of pixels to be processed by the operator to calculate the restored value of the center pixel, hence the longer the time required for the restoration of the noisy image.

(4) The NF network is trained by using very simple artificial images that can easily be generated in a computer. However, contrary to its simplicity in implementation and convenience in training, it may be used for efficiently filtering any image corrupted by impulse noise of virtually any noise density.

(5) An even better performance may be obtained by repetitive application of the proposed operator to the corrupted image. The increase in performance obtained in this way varies depending on the image properties and the density of the corrupting noise.

It should also be pointed out that a well-known disadvantage of NF approaches is their inherent "black-box" nature of operation. Indeed, NF systems are universal approximators or generalized nonlinear modeling tools that are customized for a given specific problem by training the system with an appropriate dataset [23]. Therefore, the internal structure of an NF system does not reveal any information about the actual physical processes that generated the data it models. The dependencies between the system parameters and the system responses are hidden in the system itself, and there is often no logical relationship between the meanings of the internal parameters of the NF system and the meanings of its output values.

This is also true for the NF network used as the impulse detector in the structure of the proposed impulse noise removal operator. Hence, there is no logical relationship between the internal structure of the NF impulse detector and the impulse detection process (i.e., how an impulse is detected and how this information is reflected to the shapes of the membership functions of the NF network). On the other hand, this is not a problem regarding the proposed filtering method, because the essential task of the impulse detector is the correct classification of the input pixels, and the proposed NF network successfully achieves this task.

It is concluded that the proposed operator can be used as a simple but powerful tool for efficient removal of impulse noise from digital images without distorting the useful information within the image. 


\section{REFERENCES}

[1] S. E. Umbaugh, Computer Vision and Image Processing, Prentice-Hall, Englewood Cliffs, NJ, USA, 1998.

[2] M. Sonka, V. Hlavac, and R. Boyle, Image Processing, Analysis, and Machine Vision, PWS Publishing, Pacific Grove, Calif, USA, 1999.

[3] O. Yli-Harja, J. Astola, and Y. Neuvo, "Analysis of the properties of median and weighted median filters using threshold logic and stack filter representation," IEEE Trans. Signal Processing, vol. 39, no. 2, pp. 395-410, 1991.

[4] S.-J. Ko and Y. H. Lee, "Center weighted median filters and their applications to image enhancement," IEEE Trans. Circuits and Systems, vol. 38, no. 9, pp. 984-993, 1991.

[5] B. Jeong and Y. H. Lee, "Design of weighted order statistic filters using the perceptron algorithm," IEEE Trans. Signal Processing, vol. 42, no. 11, pp. 3264-3269, 1994.

[6] T. Sun and Y. Neuvo, "Detail-preserving median based filters in image processing," Pattern Recognition Letters, vol. 15, no. 4, pp. 341-347, 1994.

[7] T. Chen and H. R. Wu, "Adaptive impulse detection using center-weighted median filters," IEEE Signal Processing Letters, vol. 8, no. 1, pp. 1-3, 2001.

[8] S. Zhang and M. A. Karim, "A new impulse detector for switching median filters," IEEE Signal Processing Letters, vol. 9, no. 11, pp. 360-363, 2002.

[9] T. Chen, K.-K. Ma, and L.-H. Chen, "Tri-state median filter for image denoising," IEEE Trans. Image Processing, vol. 8, no. 12, pp. 1834-1838, 1999.

[10] T. Chen and H. R. Wu, "Space variant median filters for the restoration of impulse noise corrupted images," IEEE Trans. on Circuits and Systems II: Analog and Digital Signal Processing, vol. 48, no. 8, pp. 784-789, 2001.

[11] Z. Wang and D. Zhang, "Progressive switching median filter for the removal of impulse noise from highly corrupted images," IEEE Trans. on Circuits and Systems II: Analog and Digital Signal Processing, vol. 46, no. 1, pp. 78-80, 1999.

[12] E. Abreu, M. Lightstone, S. K. Mitra, and K. Arakawa, "A new efficient approach for the removal of impulse noise from highly corrupted images," IEEE Trans. Image Processing, vol. 5, no. 6, pp. 1012-1025, 1996.

[13] M. S. Moore, M. Gabbouj, and S. K. Mitra, "Vector SD-ROM filter for removal of impulse noise from color images," in Proc. EURASIP Conference on DSP for Multimedia Communications and Services (ECMCS '99), Krakow, Poland, June 1999.

[14] W.-Y. Han and J.-C. Lin, "Minimum-maximum exclusive mean (MMEM) filter to remove impulse noise from highly corrupted images," Electronics Letters, vol. 33, no. 2, pp. 124125, 1997.

[15] F. Russo and G. Ramponi, "A fuzzy filter for images corrupted by impulse noise," IEEE Signal Processing Letters, vol. 3, no. 6, pp. 168-170, 1996.

[16] Y. S. Choi and R. Krishnapuram, "A robust approach to image enhancement based on fuzzy logic," IEEE Trans. Image Processing, vol. 6, no. 6, pp. 808-825, 1997.

[17] D. L. Pham and J. L. Prince, "Adaptive fuzzy segmentation of magnetic resonance images," IEEE Trans. on Medical Imaging, vol. 18, no. 9, pp. 737-752, 1999.

[18] F. Russo, "Noise removal from image data using recursive neurofuzzy filters," IEEE Trans. Instrumentation and Measurement, vol. 49, no. 2, pp. 307-314, 2000.

[19] F. Russo, "FIRE operators for image processing," Fuzzy Sets and Systems, vol. 103, no. 2, pp. 265-275, 1999.
[20] D.-C. Park and Y.-J. Woo, "Weighted centroid neural network for edge preserving image compression," IEEE Transactions on Neural Networks, vol. 12, no. 5, pp. 1134-1146, 2001.

[21] V. Boskovitz and H. Guterman, "An adaptive neuro-fuzzy system for automatic image segmentation and edge detection," IEEE Transactions on Fuzzy Systems, vol. 10, no. 2, pp. 247262, 2002.

[22] M. E. Yüksel and M. T. Yıldırım, "A simple neuro-fuzzy edge detector for digital images corrupted by impulse noise," International Journal of Electronics and Communications, vol. 58, no. 1, pp. 72-75, 2004.

[23] J.-S. R. Jang, C.-T. Sun, and E. Mizutani, Neuro-Fuzzy and Soft Computing, Prentice-Hall, Englewood Cliffs, NJ, USA, 1997.

M. Emin Yüksel received his B.S. degree in electronics and communications engineering from the Technical University of Istanbul, Istanbul, Turkey, in July 1990. In February 1991, he joined as a Research Assistant at the Department of Electronics Engineering, Erciyes University, Kayseri, Turkey. He received his M.S. and Ph.D. degrees in electronics engineering from Erciyes University in February 1993 and September

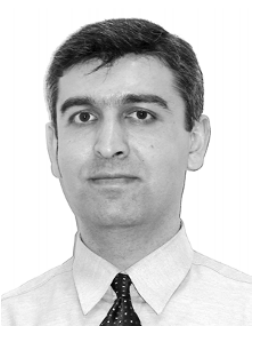
1996, respectively. Between March and December 1995, he had been an Academic Visitor to Signal Processing Section, Department of Electrical Engineering, Imperial College, London, UK, where he conducted research on time-varying parametric modeling of nonstationary signals. Currently, he is with the Department of Electronics Engineering of Erciyes University. His general research interests include signal processing, image processing, neural networks, fuzzy systems, and applications of these techniques.

Alper Baştürk received his B.S. degree in electronics engineering from Erciyes University, Kayseri, Turkey, in July 1998. He then joined the Department of Electronics Engineering of Erciyes University as a Research Assistant. He received his M.S. degree in electronics engineering from Erciyes University in August 2001. He is currently working in the same department as a Research Assistant towards the Ph.D. degree. His research areas are digital signal and image processing, neural networks, fuzzy systems, intelligent optimization, and applications of these techniques.

Erkan Beşdok received his B.S., M.S., and Ph.D. degrees in photogrammetry engineering from the Technical University of Istanbul, Istanbul, Turkey, in July 1991, July 1998 and July 2000, respectively. He worked for the private sector between 1991 and 1996. In May 1996, he joined as a Research Assistant at the Department of Photogrammetry Engineering, Erciyes University, Kayseri, Turkey. He is currently with the same

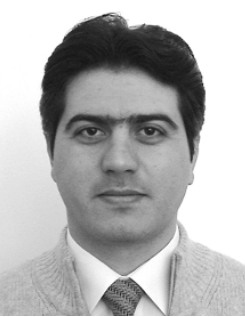
department as a full-time member of the academic staff. His current research interests include digital image processing, neural networks, fuzzy systems, and applications of these techniques in photogrammetry engineering.

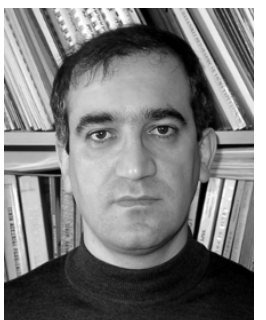

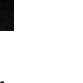

